\title{
De l'anglais dominant dans l'éducation : contributions sociolinguistiques à des réinterprétations didactiques
}

\section{Gilles Forlot}

\section{OpenEdition}

\section{Journals}

Édition électronique

URL : http://journals.openedition.org/trema/3169

DOI : 10.4000/trema.3169

ISSN : 2107-0997

Éditeur

Faculté d'Éducation de l'université de Montpellier

Édition imprimée

Date de publication : 1 novembre 2014

Pagination : 6-19

ISSN : 1167-315X

Référence électronique

Gilles Forlot, « De l'anglais dominant dans l'éducation : contributions sociolinguistiques à des réinterprétations didactiques », Tréma [En ligne], 42 | 2014, mis en ligne le 16 juin 2015, consulté le 19 avril 2019. URL : http://journals.openedition.org/trema/3169; DOI : 10.4000/trema.3169

Ce document a été généré automatiquement le 19 avril 2019

Trema 


\title{
De l'anglais dominant dans l'éducation : contributions sociolinguistiques à des réinterprétations didactiques
}

\author{
Gilles Forlot
}

\section{Introduction : les formes d'un anglais délocalisé}

1 Depuis plusieurs décennies, l'enseignement des langues a connu une évolution remarquable, notamment depuis l'avènement du communicatif et, désormais, de l'approche actionnelle (Conseil de l'Europe 2001). Pourtant, un schéma d'enseignementapprentissage plutôt traditionnel perdure, axé sur les modèles du «bain » linguistique et du locuteur natif. La langue anglaise, du fait de sa diffusion et parce que le nombre de locuteurs (ou en tout cas d'apprenants) non-natifs dépasse maintenant de loin celui des natifs (Crystal, 2011) ${ }^{1}$, a acquis un statut particulier. Les travaux et les débats entre linguistes attirent l'attention de nombre de didacticiens et pédagogues concernant l'enseignement de l'anglais à l'échelle internationale. Différentes propositions ont été faites pour que l'on questionne ce modèle du natif tout en tenant compte des particularités d'un anglais nommé tantôt langue mondiale (Brutt-Griffler 1998; 2002), tantôt langue internationale (Jenkins, 2000 ; McKay, 2002 ; 2009; Seidlhofer, 2003), tantôt lingua franca (Seidlhofer, 2009 ; 2011; Jenkins, 2007 ; Mauranen et Ranta, 2009) et les potentialités qu'il offre en termes de communication interculturelle (Meierkord, 1996; Hülmbauer, Böhringer et Seidlhofer, 2008).

Quantité de questions sociolinguistiques se posent alors à cet enseignementapprentissage de l'anglais dans le contexte mondialisé actuel. D'abord, en termes linguistiques (et, partant, didactiques), pourquoi percevoir encore les langues uniquement par le prisme de ceux qu'on appelle leurs "natifs»? Est-ce parce que l'enseignement linguistique est attaché à la langue elle-même (c'est-à-dire aux savoirs 
plutôt qu'aux savoir-faire), parce que la langue demeure pour le sens commun un des attributs de l'identité, et parce que toute hybridité serait considérée comme inférieure et illégitime (cf. Jucquois, 2003) ? Ensuite, en termes sociolinguistiques, quelles sont et comment analyser les conséquences et les profits de cette conception des choses? En quoi crée-t-elle plus ou moins de légitimité aux yeux de ceux qui parlent la langue, qui l'apprennent, qui la rejettent, etc. ? Pour finir, et il s'agit de l'objectif principal de cet article, en quoi l'anglais peut-il se concevoir comme davantage qu'une langue simplement hégémonique? Plus précisément, comment l'appropriation de l'anglais peut-il permettre de dépasser la philosophie du "tout anglais» et ouvrir sur un enseignementapprentissage des langues inscrit dans la diversité linguistique?

\section{La diffusion de l'anglais : des problématiques sociolinguistiques aux questionnements didactiques}

3 La question de la diffusion de l'anglais interroge les linguistes et les écrivains depuis longtemps, à tel point que des intellectuels, dont les dramaturges irlandais G. B. Shaw et O. Wilde, ont pu dire que l'Angleterre et l'Amérique avaient tout en commun si ce n'est la langue! Depuis les années 1930, de nombreuses tentatives de réforme de la langue anglaise ont été proposées, dont quelques unes d'illustres écrivains et grammairiens. La plupart proposent soit des opérations de simplification structurelle ou orthographique de la langue, soit une simplification de l'usage et des tournures de l'anglais par l'évitement, en particulier, d'un usage métaphorique et idiomatique de la langue ${ }^{2}$

4 Le point commun de ces approches est d'avoir repris à leur compte l'idée qu'une langue ne se parle pas nécessairement entre locuteurs d'une même variante et, a fortiori, entre natifs uniquement. Dans les années quatre-vingt-dix, certains linguistes ont posé la question fondamentale de la "propriété » (ownership) de l'anglais (Widdowson, 1994; Norton, 1997 ; Higgins, 2003). Dans un monde où il se diffuse et où on compte davantage de gens apprenant l'anglais que d'anglophones natifs, Widdowson (1994) s'est demandé qui pouvait maintenant se prévaloir de la «propriété » de l'anglais et comment cette légitimité de la propriété pouvait influer sur l'enseignement-apprentissage de cette langue. Rappelons que cette question de la propriété de la langue est souvent perçue comme centrale dans l'esprit des enseignants et des apprenants et gageons qu'elle ne se limite pas au monde anglophone.

5 Par exemple, pour la France, J.-B. Marcellesi (1979) a théorisé la tension entre ce qu'il appelle le «français institutionnel » et la variation régionale dans les conditions de l'hégémonie culturelle. Dans le monde anglophone, H. G. Widdowson (1994) propose de définir la langue standard (l'anglais en l'occurrence) comme une variété sanctionnée socialement comme étant nécessaire à l'usage institutionnel et apte à transcrire le code écrit. Il démontre que le pan oral de cette langue a moins d'importance et que le problème d'un lexique standard est largement exagéré dans la mesure où, la notion de standard engageant des formes de stabilité, la créativité lexicale de chaque communauté de locuteurs des diverses variété d'une même langue rend cette notion non fonctionnelle, non pertinente.

6 Partant de là, Widdowson montre que les anglais internationaux ont créé les conditions d'un usage institutionnel de la langue ${ }^{3}$. L'idée d'un anglais standard n'est donc pas à proprement parler scientifique, mais ancrée dans une vision élitiste et ethniciste de la 
langue dans laquelle les seuls locuteurs de la langue seraient les natifs, parce qu'ils seraient nés « avec la langue », en seraient les propriétaires.

7 Au-delà de cet héritage supposé défendu par des linguistes comme Quirk $(1985 ; 1988)$ et ceux que Pennycook $(1998 ; 2001)$ appelle les tenants de la célébration coloniale (colonial celebratory), Widdowson apporte un argument supplémentaire d'importance: les défenseurs de la langue standard, en plus d'agir comme des héritiers, se positionnent comme des gardiens, des conservateurs, des dépositaires ${ }^{4}$ de la langue et de sa norme (Sifakis, 2009). Il est donc normal que les apprenants les perçoivent aussi comme les locuteurs et, partant, les enseignants les plus légitimes...

8 La didactique institutionnelle des langues est en effet amenée, à mon sens, à envisager l'enseignement-apprentissage des langues différemment, notamment redéfinir la façon dont on perçoit l'anglais, en sortant d'un positionnement bipolaire allant de la célébration du tout anglais à la lutte effrénée contre cette langue. Pour dépasser d'un côté un projet plurilingue construit essentiellement sur la lutte contre la domination de l'anglais et d'un autre côté un attentisme innocent vis-à-vis de la progression de cette langue dans les sociétés et les systèmes éducatifs occidentaux, sans doute devrait-on réfléchir à la redéfinition de cette vision binaire - et émotive - de la question de l'anglais en France. Je reviens, dans la section qui suit, sur la manière dont certains chercheurs ont théorisé la question du développement de l'anglais à l'échelle internationale.

\section{Enseigner l'anglais de nos jours : langue étrangère, lingua franca ou langue internationale?}

9 Certains sociolinguistes et/ou pédagogues (voir par exemple Sifakis 2009) ont signalé le glissement progressif, par une modification subreptice du sigle, de l'enseignement de l'anglais (dans le cercle d'expansion de Kachru, du moins), d'un statut d' «EFL » (English as a Foreign Language, l'anglais langue étrangère) à un statut de d' "ELF » (English as a Lingua Franca, l'anglais lingua franca). Pour Seidlhofer (2005), l'anglais lingua franca doit être considéré comme une langue de contact entre locuteurs qui ne partagent ni la même langue ni la même culture. À ce titre, il participe du développement plus général de ce qu'on appelle l'anglais langue internationale ou l'anglais mondial (World English[es]), quels que soient les cercles - au sens de Kachru, 1985) - dans lesquels il progresse.

Bien que la plupart des auteurs soient d'accord sur le développement et les fonctionnements sociolinguistiques de ces formes de langue, ils ne partagent pas tous exactement la même terminologie: C. Meierkord (1996), par exemple, en situant son analyse dans le courant de la pragmatique, a perçu dans ces interactions entre non-natifs le développement d'un anglais comme médium de la communication interculturelle. J. Jenkins a quant à elle analysé en profondeur les formes de ce contact entre non-natifs et en a théorisé notamment la phonologie (cf. Jenkins, 2000), en en conservant le nom d'» anglais langue internationale ». McKay a le plus souvent aussi fait de même dans sa recherche sur le développement et l'enseignement de l'anglais dans les contextes où ce sont surtout des non-natifs qui entrent en interaction (McKay, $2002 ; 2009$ ).

11 L'intérêt de ces recherches est d'envisager un changement dans la façon dont on se représente l'anglais et les acteurs sociaux qui sont amenés à utiliser cette langue comme ressource. Par prudence, mieux vaut ne pas affirmer qu'ainsi disparaîtront les effets de domination et d'iniquité que l'anglais suscite, mais plutôt que cela permet d'entrevoir des 
solutions à une mise à disposition des ressources que constitue l'anglais de nos jours. Je ne suis sans doute pas le seul à considérer le discours de combat contre la domination linguistique comme tout aussi idéologisé (Duchêne, 2006; Cameron, 2007; Canut et al., 2009) que celui pour la diffusion des grandes langues, ainsi que le montre Pennycook (2007: 19-20) au sujet des réponses souvent nationalistes que certains sociolinguistes ou d'autres intellectuels donnent à l'expansion de l'anglais ${ }^{5}$.

\section{Des pratiques fidèles à une histoire}

Avant de se pencher plus spécifiquement sur la question de l'appropriation de l'anglais, il semble nécessaire de faire un bref détour par l'explicitation de certaines pratiques pédagogiques héritées de l'histoire sociolinguistique de la France. Inscrites dans une tradition homogénéisante, des tendances unifiantes et puristes ont rejailli sur la didactique de langues en ce sens qu'elles ont légitimé des pratiques linguistiques dans l'enseignement-apprentissage des langues s'inspirant des modèles de l'enseignement du français dit langue maternelle ou langue de scolarisation. Il y a dans ces tendances aussi bien des dimensions systémiques (les formes de la langue telle qu'on les enseigne) que des dimensions extralinguistiques de l'enseignement des langues. Par exemple, pour le cas de la France, on compte les suivantes :

\section{1. Une focalisation sur le respect scrupuleux de la norme (morphosyntaxe, orthographe...)}

13 et un travail d'apprentissage axé souvent prioritairement - ou en tout cas pendant longtemps - sur l'écrit et marqué par l'absence de prise en compte des phases transitoires inhérentes à l'appropriation d'une langue autre (cf. l'interlangue selon Selinker, 1972).

\section{2. La référence à une pratique pédagogique encore monolingue,}

par la monovalence majoritaire des enseignants de langues et par la juxtaposition cloisonnée de ces enseignements linguistiques à ceux du français langue de scolarisation, comme s'ils étaient dans une relation binaire, en face à face (Coste, 2001 ; Ducancel \& Simon, 2004).

\section{3. La recherche d'éléments unificateurs de la langue.}

Il s'agit ici d'une orientation monolingue qui rejaillit sur la simplification de la perception de ce qu'est une langue, dans laquelle il y a très peu de variation, des pratiques dialectales occultées, des croisements - pourtant existants - rejetés ou stigmatisés. Cette phrase, dénichée dans le rapport de l'agrégation externe de portugais de 2004, illustre bien cette compartimentation linguistique :

«Rappelons que la norme portugaise et la norme brésilienne sont admises, mais il ne doit pas y avoir d'interférences entre les deux " (CNDP, $2004: 29)$.

Les rapports des concours de l'enseignement (CAPES et agrégation) pour l'anglais mettent eux aussi en valeur la nécessité que les candidats se conforment à l'une des deux variantes préférées: le Southern British English, l'anglais de l'Angleterre parlé au sud des Midlands, et le General American, l'anglais américain standard, issu des parlers du Midwest 
et excluant donc les formes accentuelles trop marquées, comme celles du sud des ÉtatsUnis ou de New York. La consigne est même la suivante pour l'épreuve de phonologie :

"Regardless of the origin of the text, candidates are free to base their transcriptions on either Southern British English (RP / BBC English) or on General American, to the exclusion of any other variety of English" (Cité dans Jobert, 2009 : 96)

17 Jobert indique aussi qu'à l'épreuve de phonologie de l'agrégation d'anglais, les deux seuls dictionnaires spécialisés autorisés sont le Longman Pronouncing Dictionary et le English Pronouncing Dictionary, deux dictionnaires ayant pour référence l'anglais britannique standard (Jobert, 2009). Inutile alors de parler ici des autres formes d'anglais "natif ", celle(s) des Canadiens, proche(s) du General American ${ }^{7}$, celle(s) des Australiens, des NéoZélandais, des Sud-Africains, ni celle(s) du Hiberno-English (l'anglais d'Irlande). Ainsi, restreint-on, finalement de façon assez contradictoire, la légitimité du "natif » à quelques parlers de grande diffusion, balayant en passant la complexité de ces derniers.

\section{4. La simplification de réalités sociolinguistiques complexes,}

par exemple celles qui concernent l'enseignement de l'arabe (la question étant ici, quel arabe apprendre, entre le littéral, le classique et les dialectaux, alors qu'ils diffèrent notablement ; voir, pour la France, Caubet, 2003 ou Barontini, 2007) ? Se pose aussi la question de la complexité du continuum dialectal de nombreuses langues régionales de France, qu'elles soient enseignées sous des formes supposément standardisées (occitan, breton, etc.) ou perçues comme polynomiques (entre autres le corse et le provençal), ou encore non enseignées officiellement quoique assez présentes dans les pratiques quotidiennes (les parlers picards, par exemple).

Ces questions sont complexes, car au-delà de questions d'apprentissage, ce sont des problématiques d'appropriation qui entrent en jeu, engageant avec elles des dimensions identitaires nettement perceptibles. Dans un corpus recueilli en France auprès de professeurs des écoles en formation (Forlot, 2006; 2009), les questions linguistiques se trouvent souvent placées au cœur de problématiques de réussite scolaire et de dimensions « essentialisantes » d'insertion et d'identité.

\section{Un projet didactique de l'émancipation}

Les questions d'appropriation des langues étant fortement liées à des trajectoires (physiques ou intellectuelles) individuelles et construites socialement dans l'enseignement traditionnel par la prégnance des représentations tant autour des objets à apprendre et à enseigner qu'autour des individus et des institutions concernés par ces appropriations, il semble désormais qu'une réflexion innovante autour de la pédagogie de l'anglais inscrite dans un projet éducatif pluriel (Coste, 2013) soit à l'ordre du jour.

\section{1. Sortir des cercles kachruviens}

Une démarche didactique renouvelée appelle donc un affranchissement du modèle du « cercle intérieur » (tel que défini par Kachru, 1985), c'est-à-dire de l'anglais des espaces où la langue majoritaire - la L1, dite langue maternelle - est l'anglais, même si on a vu supra que généralement, seuls l'anglais britannique standard et l'américain standard étaient véritablement légitimés (en tout cas en France) comme formes linguistiques à 
enseigner. Bien entendu, il s'agit ici de simplifications pour les nécessités de la démonstration, attendu que d'une part, dans ces pays, nombre de locuteurs n'ont pas l'anglais comme langue 1 , ou sont bi/plurilingues, et que d'autre part, dans des pays du cercle extérieur (Inde, Pakistan, Jamaïque, Philippines...) existe une classe dominante éduquée en anglais et maîtrisant cette langue au même niveau que les natifs. D'autre part, rappelons que cette considération sur les pays anglophones, si l'on reprend la façon dont Pennycook (2007) et Blommaert (2010) abordent les choses, ignore largement la "nativisation" de ces morceaux d'anglais qui circulent au gré des contacts transculturels. Mais pour l'intérêt de la démonstration, poursuivons avec la question de l'enseignement de l'anglais et de ses rapports avec le « cercle intérieur » de l'anglais.

\section{2. La langue comme instrument : communication et enseignement}

Pour les apprenants, s'affranchir, s'émanciper du " cercle intérieur », c'est considérer que l'on construit ses apprentissages dans l'optique d'une appropriation de l'anglais de communication entre non-natifs. Il y a là, j'en conviens, une dimension instrumentale des langues et une marchandification ${ }^{8}$ de celles-ci. Dans les discours d'enseignants et d'étudiants (Forlot, 2006), l'anglais est perçu comme une langue utile, indispensable, incontournable, en ce sens que ses locuteurs ou ses apprenants considèrent qu'elle permet d'un côté d'entrer en contact avec l'» autre » au-delà des frontières et des aires linguistiques et d'un autre côté d'avoir accès à une grande partie de la communication et de l'information en ligne (sites web, e-mails) ainsi que d'écrits scientifiques publiés en anglais.

23 Pour les enseignants, s'affranchir du modèle du natif, c'est regagner des formes de légitimité que la comparaison systématique avec le modèle du natif met à mal (cf. Seidlhofer, 2003 ; Sifakis, 2009). Passer de l'anglais langue étrangère (EFL) à l'anglais lingua franca (ELF) permet de reconstruire une place légitime à l'enseignant de la langue plutôt qu'au locuteur de la langue et ainsi de « dénativiser » la langue (Castellotti, 2011). L'enseignant, en sortant du modèle du cercle intérieur, quitte son rôle de gardien et d'héritier d'un modèle supposément intangible (le concept de custodian avancé par Widdowson 1994) pour embrasser un "statut de passeur, d'intermédiaire, de médiateur " (Castellotti \& Moore, 2002 ; cf. aussi Coste, 2001).

\section{3. L'anglais : propriété et légitimité}

Dans l'enseignement des langues conçu de cette façon, la langue n'est plus construite comme un objet figé porteur d'une valeur inhérente (Canut, 2010), mais une ressource mobilisable et actualisable dans l'interaction sociale (cf. Boutet \& Heller, 2007; Blommaert, 2010). L'anglais, puisque c'est le cas qui nous intéresse ici, langue qui s'instancie de nos jours le plus fréquemment entre non-natifs, est voué à sortir du cercle intérieur et, à terme, à se libérer de la seule légitimité du natif.

En France, ces discussions sont peu connues. On considère sans doute comme évident que l'anglais «appartient» (cf. le concept de propriété ou d'appartenance - en anglais ownership - discuté plus haut) au monde anglophone, et parler anglais doit tendre vers les formes linguistiques du natif. Lorsque j'ai l'occasion de présenter ces arguments, qui pour l'essentiel participent d'une démarche sociolinguistique interventionniste en faveur de 
l'appropriation d'un anglais émancipé du cercle intérieur, les critiques sont nombreuses : on y perçoit souvent du laxisme, un risque d'appauvrissement de la langue, un « anglais OGM » et la porte ouverte au tout-anglais.

D'autre part - mais j'ai peu de place pour entrer ici dans les détails - cela pose d'importantes questions épistémologiques en didactique des langues : on s'éloignerait en effet de la vision de l'indissociabilité du couple «langue-culture» (cf. Byram, 1992; Zarate, 1993 ; Beacco, 2000), car dans ce cas, la langue serait « déculturalisée ", même si un flou subsiste à mon sens sur la façon dont est pris en charge le concept de culture dans l'enseignement-apprentissage des langues à l'heure actuelle.

\section{L'anglais au cœur de la didactique des langues?}

\section{I. Les enseignants d'anglais, passeurs de langues?}

À l'école secondaire française, on considère parfois que le centre névralgique, pour ainsi dire, de tous les apprentissages langagiers à l'école, c'est le professeur de français (langue de scolarisation). On pourrait toutefois avancer qu'au contraire, les professeurs de français ne se perçoivent peut-être pas (ou pas encore) comme des professeurs de langue, mais de lettres, terme générique ancrant encore leur fonction dans le littéraire, les belleslettres et tout ce qu'elles charrient de grammaire normative (cf. Bertucci \& Corblin, 2004).

S'il est vrai que les professeurs de français ont toute leur place dans les appropriations langagières prises dans un sens global et devraient en devenir pour ainsi dire le moteur, on rappellera que pour des raisons sans doute idéologiques - peur du tout-anglais, domination d'un modèle américain, défense d'une exception culturelle française... -, on oublie que les enseignants qui se trouvent véritablement à la croisée de tous les chemins de l'altérité linguistique, de l'école à l'université, ce sont ceux qui enseignent l'anglais.

Certains linguistes s'intéressent à la place de l'anglais en des termes linguistiques et dans leurs applications pédagogiques (Robert, 2008 ; Castagne, 2008 ; Neuner, 2004...). Il s'agit toutefois aussi d'une question tout à fait sociolinguistique. En effet, la problématique des représentations en milieu scolaire est cruciale, car les apprenants sont entourés de «discours circulants» (Heller, 2002) qui émanent de divers locuteurs ou groupes de locuteurs détenant des formes de pouvoir ou d'autorité, qu'elles soient personnelles, scolaires ou médiatiques : la famille proche, les enseignants, les inspecteurs, les experts ou en tous cas les personnes présentées comme telles - invités dans la presse ou les politiques lançant débats et polémiques sur le langue, son enseignement, sa qualité, etc.

De plus, un autre volet sociolinguistique s'ouvre dans le fait que l'anglais, comme on l'a dit, ne peut plus vraiment être considéré comme une langue comme les autres. Ainsi, le détour didactique proposé ici oblige à prendre tout autant le statut (status) que le corpus de l'anglais en considération, et ainsi de travailler sur une langue dominante pour la déconstruire comme outil et ressource d'aide à l'apprentissage des langues. Dit autrement, l'hégémonie de l'anglais est pour ainsi dire nécessaire pour les propositions faites ici: il s'agit de tirer profit de la présence de l'anglais pour construire des apprentissages plurilingues. Quelques chercheurs, dans d'autres optiques épistémologiques que les miennes, ont travaillé aussi sur cette question : par exemple, M.C. Deyrich et S. Olivé (2009) ont travaillé sur les ponts entre anglais et français dans l'approche de la narration et M. Kervran a consacré sa thèse (cf. Kervran, 2008 et ses 
travaux ultérieurs) à un glissement d'une didactique de l'anglais à celle d'une éducation langagière plurielle.

\section{2. L'anglais : capital et accélérateur}

31 Résumées brièvement, les potentialités didactiques présentées ici partent du constat que l'anglais, tout en restant une langue germanique, peut aussi être considérée comme une langue en partie romane, du fait d'un lexique et d'une syntaxe plus ou moins fortement influencés par les invasions romaines et normandes de l'Angleterre (Strang, 1990 ; Crépin, 1994). En effet, ses caractéristiques linguistiques étant marquées par des formes d'hybridité, l'anglais paraît immédiatement familier à de nombreux locuteurs. Les apprenants de diverses langues germaniques ou latines qui auront aussi étudié l'anglais pourront avoir un sentiment de "déjà-appris » ou de "déjà-compris », en somme une sorte d'écho dans certains fonctionnements linguistiques des langues qu'ils apprennent.

Les exemples de la parenté lexicale sont bien connues (cf. Walter, 2001), même si certains relèvent plus des cognats (Crépin, 2007) que des transférences directes. J'ai présenté dans d'autres travaux quelques dimensions linguistiques qui me semblent pertinentes à un changement de statut de l'anglais dans l'enseignement scolaire (Forlot, 2012; à paraître $a$; à paraître b).

Partant de ces constats structurels et du fait que la demande sociale et économique pour cette langue est très forte, les acteurs du champ éducatif pourraient, plutôt que de tenter - vainement - d'occulter l'anglais par des mesures coercitives de politique linguistique, au contraire le faire sortir au grand jour et en faire une langue passerelle vers les autres langues et la diversité linguistique. Bien sûr, ce projet n'a de sens que si la gestion de l'anglais langue d'éducation est contrôlée et planifiée: il ne s'agit pas d'élargir le boulevard de l'anglais, mais de faire de cette langue un accélérateur de plurilinguisme.

\section{Conclusion et ouverture : l'anglais comme capital, pour qui, pourquoi et à quelles conditions?}

On pense souvent à l'anglais comme capital économique, comme clé du succès professionnel, comme porte d'accès au commerce, à la finance, etc. Soit, cela relève d'une évidence à laquelle la loi de l'économie mondialisée nous a peu à peu habitués. Néanmoins, je le conçois aussi comme un capital d'un autre ordre. Capitaliser des connaissances linguistiques pour en acquérir d'autres est un processus bien connu des chercheurs: nombre de travaux ont montré que les apprenants tirent profit, même inconsciemment, des autres langues qu'ils ont apprises antérieurement ou qu'ils apprennent simultanément. On sait que beaucoup d'apprenants recherchent des indices de proximité ou de transférabilité (Hammarberg, 2001; De Angelis, 2005) et qu'ils s'appuient souvent davantage sur les langues secondes apprises plutôt que sur leur langue maternelle (Bono, 2008).

En France et en Europe en général, l'utilisation de l'anglais comme langue passerelle se prête particulièrement bien à ces manipulations. La proximité lexicale entre l'anglais et le français, souvent l'objet de crainte d'interférences (Dabène, 1996 ; Castellotti, 2001), doit être mise à profit justement pour attirer l'attention sur les différences. Des expériences menées il y a quelques années (Forlot \& Beaucamp, 2008 ; Beaucamp, 2008) ont montré les 
bienfaits de la contrastivité phonologique et prosodique - sous forme de jeux d'écoute, par exemple (cf. Hawkins, 1987) - entre des mots transparents de l'anglais et du français dans une classe de CM2.

Sur un plan plus particulièrement didactique, un tel projet incite les enseignants à sortir de tendances à l'isolement linguistique. Sans être ni tenter de devenir polyglotte, l'enseignant d'anglais peut être attentif aux formes de proximité des langues et/ou aux aides potentielles qu'elles peuvent apporter aux élèves. Ceci tient du développement d'une pédagogie des possibles (Hélot \& Ó Laoire, 2011).

Il participe aussi de la construction de compétences et au développement de réflexes métalinguistiques chez les apprenants, encourageant ainsi certaines formes d'autonomie dans l'apprentissage, tout en permettant à la langue anglaise d'assumer une fonction de soutien au développement du plurilinguisme. Ceci pourrait mener aussi, à l'école, à un dépassement de la représentation dominante d'une nécessité purement utilitaire de l'anglais.

Sans tomber dans le piège de la naïveté et de l'angélisme, on peut tout de même promouvoir le développement d'une " philosophie » de la décompartimentation scolaire, non seulement pour que les langues pénètrent dans les autres parties du curriculum, mais aussi pour qu'elles interagissent entre elles en vue d'apprentissages facilités par les principes du transfert des savoirs linguistiques et des savoir-faire langagiers. On peut espérer du français, langue de scolarisation, mais aussi de l'anglais, langue hypercentrale, qu'ils puissent jouer ces rôles de pivots et de passerelles.

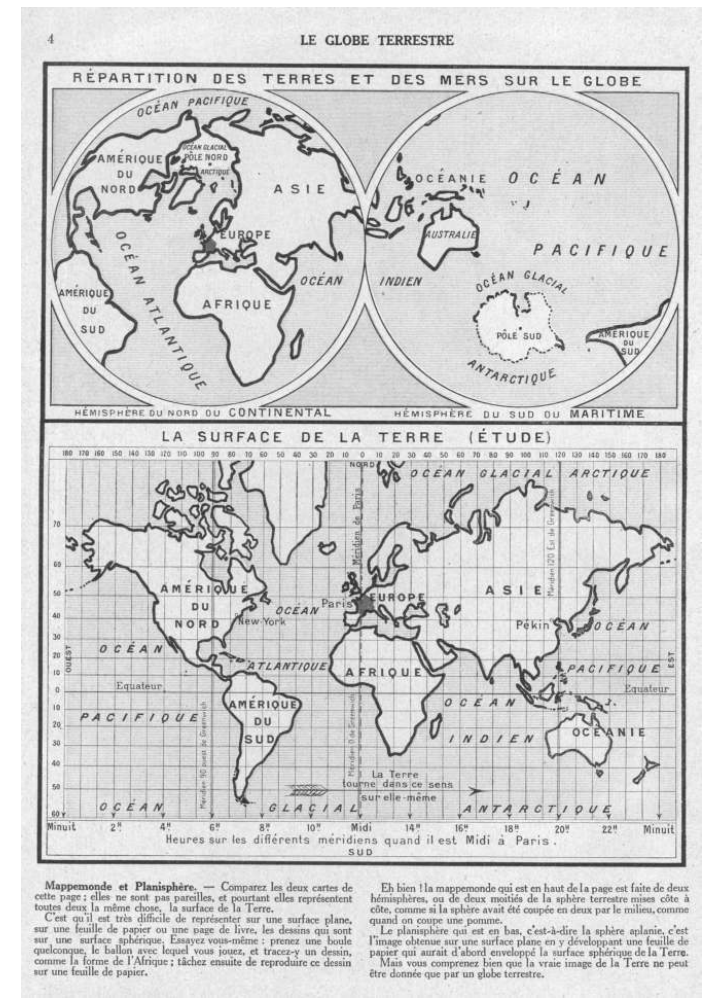

(1923). Cours complet de géographie, cours moyen, le globe terrestre, p. 4. Cedrhe : 5478 


\section{BIBLIOGRAPHIE}

Barontini, A. (2007). « Valorisation des langues vivantes en France : le cas de l'arabe maghrébin », Le français aujourd'hui 158, pp. 20-27.

Beacco, J.-C. (2000). Les dimensions culturelles des enseignements de langue. Paris : Hachette.

Beaucamp, J. (2008). « Enseigner une langue vivante étrangère à l'école (cycle 3) : opportunité d'un début de réflexion métaphonologique et métalinguistique », Les Cahiers de l'ACEDLE 3, pp. $15-39$.

Bertucci, M.-M \& Corblin, C. (dir.) (2004). Quel français à l'école ? Les programmes de français face à la diversité linguistique, Paris : L'Harmattan.

Blommaert, J. (2010). The Sociolinguistics of Globalization, Cambridge : CUP.

Bono, M. (2008). « Influences interlinguistiques dans l'apprentissage d'une L3 : quand les langues secondes l'emportent sur la langue première ", In Moore, D. et Castellotti, V. (dir.), La compétence plurilingue : regards francophones (pp.147-166). Berne : Peter Lang.

Boutet, J. \& Heller, M. (2007). « Enjeux sociaux de la sociolinguistique : pour une sociolinguistique critique ", Langage et Société 121-122, pp.305-318.

Brutt-Griffler, J. (2002). World English. A Study of its Development, Clevedon: Multilingual Matters.

Byram, M. (1992). Culture et éducation en langue étrangère, Paris : Didier/Hatier.

Cameron, D. (2010). «Globish: How the English Language Became the World's Language by Robert McCrum», The Guardian, 05/06/2010.

Cameron, D. (2007). «Language endangerment and verbal hygiene: History, morality and politics», In Duchêne, V. \& Heller, M. (dir.), Discourses of Endangerment. Interest and Ideology in the Defense of Languages (pp.268-285). London : Continuum.

Cameron, D. (2000). «Styling the worker: Gender and the commodification of language in the globalised service economy», Journal of Sociolinguistics 4/3, pp.323-347.

Canut, C. (2010). Une langue sans qualité, Limoges : Éditions Lambert-Lucas.

Canut, C., Bodourova, D. \& Caroli, E. (2009). Langues à l'encan, essai sur la capitalisation linguistique en Europe, Paris : Michel Houdiard.

Castagne, E. (2008). « Les langues anglaise et française : amies ou ennemies ? ", Études de Linguistique Appliquée 149, pp.31-43.

Castellotti, V. (2011). « Natif, non-natif ou plurilingue : dénativiser l'enseignement des langues? ». In Dervin, F. et Badrinathan, V. (dir.), L'enseignant non natif: Identités et légitimité dans l'enseignement-apprentissage des langues étrangères (pp.29-50). Fernelmont : Éditions modulaires européennes.

Castellotti, V. (2001). La langue maternelle en classe de langue étrangère, Paris : CLE International.

Castellotti, V. et Moore, D. (2002). Représentations sociales des langues et enseignement, Strasbourg : Conseil de l'Europe. 
Caubet, D. (2003). « La reconnaissance de l'arabe 'dialectal' en France : un parcours sinueux », In Lentin, J. et Lonnet, A. (dir.), Mélanges à Daniel Cohen ; Études sur le langage, les langues, les dialectes, les littératures, offertes par ses élèves, ses collègues, ses amis, présentées à l'occasion de son anniversaire, Paris : Maisonneuve.

Chotiner, I. (2010). «Globish for beginners. If the whole world speaks English, will it still be English?» The New Yorker, 31/05/2010.

Conseil de l'Europe (2001). Cadre européen commun de référence pour les langues : Apprendre, enseigner, évaluer, Paris : Didier.

Coste, D. (dir.) (2013). Les langues au cœur de l'éducation. Principes, pratiques, propositions. (Association pour le développement de l'éducation bi/plurilingue), Fernelmont : Éditions modulaires européennes.

Coste, D. (2001). « De plus d'une langue à d'autres encore. Penser les compétences plurilingues ?» In Casttelloti, V. (dir.), D'une langue à d'autres : Pratiques et représentations (pp.191-202). Rouen : Presses Universitaires de Rouen.

Crepin, A. (2007). « Le plurilinguisme de l'Angleterre médiévale », Carnets d'Ateliers de Sociolinguistique 2, pp.28-44.

Crepin, A. (1994). Deux mille ans de langue anglaise, Paris : Nathan.

Crystal, D. (2011) [2e éd.], The English Language: A Guided Tour of the Language, London: Penguin.

Dabène, L. (1996). « Pour une contrastivité revisitée », Études de Linguistique Appliquée 104, pp.393-400.

De Angelis, G. et Selinker, L. (2001). «Interlanguage Transfer and Competing Linguistic Systems in the Multilingual Mind», In Cenoz, J., Hufeisen, B. et Jessner, U. (dir.), Cross-linguistic Influence in Third Language Acquisition : Psycholinguistic Perspectives (pp.42-58). Clevedon: Multilingual Matters.

Deyrich, M.-C. et Olive S. (2009). « Des passerelles entre les apprentissages langagiers et culturels à l'école : du français à l'anglais et réciproquement ", In Forlot, G. (dir.), L'anglais et le plurilinguisme. Pour une didactique des contacts et des passerelles linguistiques (pp.59-77). Paris : L'Harmattan.

Ducancel, G. et Simon D.-L. (2004). « De deux monolinguismes vers une éducation plurilingue », Repères 29, pp.3-21.

Duchêne, A. (2006). « 'Dans les États où il existe des minorités...' : les conditions de production institutionnelle, discursive et idéologique d'un article de loi aux Nations Unies », Semen 21 ( http://semen.revues.org/1977).

Forlot, G. (à paraître a), « Hégémonique, hypercentral, natif, international, passerelle... Que faire de l'anglais dans les espaces éducatifs ? ", In Causa, M. , Galligani, S. et Vlad, M. (dir.), Les enseignants de langues face au changement : besoins en formation et dispositifs en contexte. Paris : Riveneuve.

Forlot, G. (à paraître b), « La place de l'anglais dans l'éducation. Vers des pratiques complexes et plurielles d'enseignement-apprentissage des langues ». In Revue japonaise de didactique du français $n^{\circ} 8$.

Forlot, G. (2012). « Vers une formation au plurilinguisme à l'école française. De l'état des lieux à la réinterprétation des approches didactiques 'mono' des Instructions Officielles », In Balsiger, C. , Betrix, D. , De Pietro, J.-F. et Perregaux, C. (dir.), Éveil aux langues et approches plurielles. De la formation des enseignants aux pratiques de classe (pp.57-73). Paris : L'Harmattan. 
Forlot, G. (2009). « Vers la déconstruction d'un apprentissage idéologique : des "représentationsobstacles" dans l'enseignement des langues en France », Les Cahiers de l'ACEDLE 6/1, pp.65-91.

Forlot, G. (2006). « Des pratiques aux stéréotypes sociolinguistiques d'étudiants-professeurs. Résultats préliminaires d'une enquête et pistes de recherche ", Spirale 38, pp.123-140.

Forlot, G. et Beaucamp, J. (2008). « Heurs et malheurs de la proximité linguistique dans l'enseignement de l'anglais au primaire », Études de linguistique appliquée 148, pp.77-92.

Grzega, J. (2005). «Towards Global English via Basic Global English (BGE): Socioeconomic and Pedagogic Ideas for a European and Global Language (with Didactic Examples for Native Speakers of German», Journal for EuroLinguistiX 2, 65-164.

Hammarberg, B. (2001). «Roles of L1 and L2 in L3 production and acquisition», In Cenoz, J., Hufeisen, B. et Jessner, U. (dir.), Cross-linguistic Influence in Third Language Acquisition: Psycholinguistic Perspectives (pp.21-41). Clevedon: Multilingual Matters.

Hawkins, E. (1987). Modern languages in the curriculum, Cambridge : CUP.

Heller, M. (2010). «The commodification of Language», Annual review of Anthropology 39, pp.101-114.

Heller, M. (2002). Éléments d'une sociolinguistique critique, Paris : Didier.

Hélot, C. et Ó Laoire, M. (dir.) (2011). «Introduction : From language education policy to a pedagogy of the possible ", In Hélot, C. et Laoire, M. Ó (dir.), Language Policy for the Multilingual Classroom. Pedagogy of the Possible (pp.11-25). Bristol : Multilingual Matters.

Higgins, C. (2003). «'Ownership’ of English in the Outer Circle : An Alternative to the NS-NNS Dichotomy", TESOL Quarterly 37/4, pp.615-644.

Hülmbauer, C., Böhringer, H. et Seidlhofer, B. (2008). «Introducing English as a lingua franca (ELF): Precursor and partner in intercultural communication», Synergies Europe 3, pp.25-36.

Jenkins, J. (2007). English as a Lingua Franca : Attitude and Identity, Oxford : OUP.

Jenkins, J. (2000). The Phonology of English as an International Language, Oxford : OUP.

Jobert, M. (2009). « Le ‘General American' à l'épreuve de phonologie de l'agrégation », Cercles ( occasional papers 2009), pp.95-116.

Jucquois, G. (2003). « Hybridité », In Ferreol, G. \& Jucquois, G. (dir.), Dictionnaire de l'altérité et des relations interculturelles (pp.147-153). Paris : Armand Colin.

Kachru, B. (1985). «Standards, codification, and sociolinguistic realism: the English language in the Outer Circle», In Quirk, R. \& Widdowson, H.G. (dir.), English in the World: Teaching and Learning the Language and Literatures (pp.11-30). Cambridge : CUP.

Kervran, M. (2008). Apprentissage de l'anglais et « éveil aux langues » à l'école primaire : développement et transfert de compétences métalinguistiques dans le cadre d'une didactique intégrée, Thèse de doctorat, Université du Maine-Le Mans.

Marcellesi, J.-B. (1979). « Quelques problèmes de l'hégémonie culturelle en France : langue nationale et langues régionales », International Journal of the Sociology of Language 21, pp.63-80

Mauranen, A. \& Ranta, E. (dir.) (2009). English as a Lingua Franca: Studies and Findings, Newcastle upon Tyne : Cambridge Scholars Publishing.

McCrum, R. (2010). Globish: How the English Language Became the World's Language, New York : W.W. Norton. 
McCrum, R. (2006). «So, what's this Globish revolution?» The Observer, 03/12/2006.

McKay, S. L. (2009). «English as an International Language: Where we are and where we need to go», Journal of English as an International Language 5, pp.27-53.

McKay, S. L. (2002). Teaching English as an International Language, Oxford: OUP.

Meierkord, C. (1996). Englisch als Medium der interkulturellen Kommunikation. Untersuchungen zum non-native/non-native speaker Diskurs, Frankfurt am Main: Peter Lang.

Nerrière, J.-P. (2004). Don't speak English, parlez Globish ! Paris: Eyrolles.

Neuner, G. (2004). « Le concept de plurilinguisme et la didactique de langues tertiaires ». In Hufeisen, B. \& Neuner, G. (dir), Le concept de plurilinguisme : Apprentissage d'une langue tertiaire L'allemand après l'anglais (pp.13-35). Strasbourg : Conseil de l'Europe-ECML/CELV.

Norton, B. (1997). «Language, Identity, and the Ownership of English», TESOL Quarterly 31/3 ('Language and Identity'), pp.409-429.

Pennycook, A. (2007). Global Englishes and Transcultural Flows, Oxford: Routledge.

Pennycook, A. (2001). Critical Applied Linguistics. A Critical Introduction, Mahwah: Lawrence Erlbaum.

Pennycook, A. (1998). English and the Discourses of Colonialism, London: Routledge.

Quirk, R. (1988). «The Question of Standards in the International Use of English», In Lowenberg, P. (dir.), Language Spread and Language Policy: Issues, Implications and Case Studies (pp.229-241). Washington : Georgetown University Press.

Quirk, R. (1985). «The English Language in a Global Context», In Quirk, R. \& Widdowson, H. G. (dir.), English in the World: Teaching and Learning the Language and Literatures (pp.1-6). Cambridge : CUP.

Robert, J.-M. (2008). «L'anglais comme langue proche du français ? », Études de Linguistique Appliquée 149, 9-20.

Seidlhofer, B. (2011). Understanding English as a Lingua Franca, Oxford: OUP.

Seidlhofer, B. (2009). «Common ground and different realities: World Englishes and English as a Lingua Franca», World Englishes 28/2, 236-245.

Seidlhofer, B. (2005). «English as a lingua franca», ELT Journal 59/4, 339-341.

Seidlhofer, B. (2003). Autour du concept d'anglais international : de « l'anglais authentique » à «l'anglais réaliste»? Strasbourg : Conseil de l'Europe.

Sifakis, N. (2009). «Challenges in teaching ELF in the periphery: the Greek context», ELT Journal $63 / 3,230-237$.

Strang, B. M. H. (1990) [1970], History of the English Language, London: Routledge.

Walter, H. (2001). Honni soit qui mal y pense, Paris : Laffont.

Widdowson, H. G. (1998). «EIL: Squaring the circles. A reply», World Englishes 17/3, 397-401.

Widdowson, H. G. (1994). «The Ownership of English», TESOL Quarterly 28/2, 377-389.

Zarate, G. (1993). Représentations de l'étranger et didactique des langues, Paris : Didier. 


\section{NOTES}

1. Sous le règne de la Reine Élisabeth Ie (seconde moitié du XVIe siècle), le nombre de locuteurs de l'anglais se situait entre 5 et 7 millions de personnes. Au début du règne de la deuxième reine Élisabeth (1952), on estimait ce nombre à 250 millions. De nos jours, on considère qu'il y a environ 400 millions de locuteurs natifs et de 500 millions à un milliard de locuteurs de l'anglais non-natifs (Crystal, $2011: 1-3$ ).

2. On notera par exemple le Basic English de C. K. Ogden, revu et popularisé par le romancier H. G. Wells, puis la naissance du Plain English de E. Gowers et, à la même époque, d'un anglais simplifié destiné à la propagande radiodiffusée américaine en pleine guerre froide, le Special English de la radio Voice of America (1959). Plus récemment, le concept de Globish s'est largement diffusé sous l'impulsion d'un homme d'affaire français, Jean-Paul Nerrière (2004) et a suscité un riche débat journalistique qui dure encore (McCrum, 2006; 2010 ; Chotiner, 2010 ; Allen, 2011), auquel des sociolinguistes de renom ont d'ailleurs pris part (Cameron, 2010). Le linguiste allemand J. Grzega (2005) a émis l'idée que l'anglais, à condition d'en faire un usage simplifié, pouvait permettre de mettre en œuvre et de développer des compétences d'intercompréhension utile dans différents contextes internationaux.

3. On retrouve là aussi les arguments des théoriciens de l'indigénisation / nativisation comme $\mathrm{B}$. Kachru ou S. Mufwene, qui font de ces variantes internationales, de leurs lexiques par exemple, des langues standard en elles-mêmes, si l'on accepte de retenir ce mot.

4. Le mot anglais custodian, employé par Widdowson (1994), englobe tout cela : héritier, gardien, dépositaire, ou conservateur.

5. Pennycook cite notamment le livre de $\mathrm{C}$. Hagège Combat pour le français. Au nom de la diversité des langues (2006), dans lequel la diversité est construite, de façon nationale/nationaliste, dans la promotion d'une langue française qui serait la réponse au développement global de l'anglais (Pennycook, 2007: 19-20).

6. «Quelle que soit l'origine du texte, les candidats ont la liberté de baser leur transcriptions soit sur l'anglais britannique du sud (la prononciation de prestige dite Received Pronunciation / l'anglais de la $\mathrm{BBC}$ ), soit sur l'anglais américain standard, dit General American, à l'exclusion de toute autre variété d'anglais » (ma traduction).

7. Néanmoins, les Anglo-Canadiens n'appliquent pas l'ensemble des modifications orthographiques américaines proposées à la fin du XVIIIe siècle par Noah Webster, ce qui montre ici encore que les frontières entre formes linguistiques ne sont que des artifices idéologiques.

8. Au sens du mot anglais commodification (Cameron, 2000 ; Heller, 2010), qui renvoie au processus de transformation d'un objet (la langue, ici) en une marchandise (commodity) dont on peut tirer des profits.

\section{RÉSUMÉS}

Cet article s'intéresse aux dimensions sociolinguistiques de la domination de l'anglais dans l'enseignement-apprentissage des langues. En réinterprétant la place de l'anglais dans les milieux éducatifs et en dépassant la représentation binaire et simplificatrice envisageant l'anglais tantôt uniquement comme une langue hégémonique, tantôt seulement comme une 
langue incontournable, l'article propose de revisiter les schémas classiques de l'enseignement des langues - tels que le modèle du natif ou la vision homogénéisante des langues - en considérant notamment l'anglais comme langue pivot, potentiellement à la source d'appropriations plurilingues. L'hypothèse de travail de cet article est donc que loin de contribuer à construire un boulevard au tout-anglais dans l'éducation, la mise au centre de cette langue peut se concevoir aussi comme un accélérateur de plurilinguisme.

The article tackles some sociolinguistic aspects of the domination of English in language learning and teaching. After reinterpreting the place of English in educational settings as well as going beyond the binary and simplifying image of English as either a merely hegemonic or unavoidable language, what is proposed here is to revisit the classical pedagogical approaches to language teaching, such as the native language model or the homogenizing perception we have of languages, and to consider English as a pivot language which could potentially serve as a source of multilingual appropriations. The working hypothesis of this article is therefore that English does not necessarily open up to dominance, but can be conceived as a booster of multilingualism.

\section{INDEX}

Mots-clés : anglais, domination, éducation, plurilinguisme

Keywords : dominance, education, English, multilingualism

\section{AUTEUR}

\section{GILLES FORLOT}

Maître de conférences HDR INALCO Sorbonne Paris Cité, EA 4514 PLIDAM 